\title{
Narrative Navigation: Visualizing Story Order and Locations in Augmented Reality
}

\author{
Qimo Zhang \\ Computer Science Department \\ University of Waikato \\ New Zealand \\ qz116@students.waikato.ac.nz
}

\author{
Annika Hinze \\ Computer Science Department \\ University of Waikato \\ New Zealand \\ hinze@waikato.ac.nz
}

\author{
Nicholas Vanderschantz \\ Computer Science Department \\ University of Waikato \\ New Zealand \\ vtwoz@waikato.ac.nz
}

\begin{abstract}
Narrative Navigation connects mobile storytelling with geolocations and Augmented Reality (AR). Narrative Navigation is designed to help users better understand a story. In this paper, we introduce our mobile app prototype that guides users to the physical locations of a story using AR flags. We indicate not just story locations and the user's distance to those story points, but also show the flow of a story through its geo-locations. The prototype thus provides users with a new way to immerse themselves in a locationbased story.
\end{abstract}

\section{CCS CONCEPTS}

- Human-centered Computing $\rightarrow$ Mixed / Augmented Reality

- Information Systems $\rightarrow$ Location Based Services

\section{KEYWORDS}

Augmented Reality; Storytelling; Location-based Services; Mobile Applications

\section{INTRODUCTION}

Mobile location-based services (LBS) help to tell stories about locations, such as in location-based audiobooks [1]. Location-based storytelling connects stories to the physical world. Yet the appropriate techniques to indicate the story elements and guide the users to the next part of the story are still to be determined. Wither et al. [2] proposed that the gap between the real world and the story world be filled with mixed reality technology. An augmented location-based experience can be added to the narrative to make the story presentation more immersive. Combining location-based data with Augmented Reality (AR) allows the readers to be more deeply integrated into the story in multiple ways.

Our research focuses on the question: How can location-based data be visualized using augmented reality in a mobile story-telling

Permission to make digital or hard copies of part or all of this work for personal or classroom use is granted without fee provided that copies are not made or distributed for profit or commercial advantage and that copies bear this notice and the full citation on the first page. Copyrights for third-party components of this work must be honored. For all other uses, contact the Owner/Author. $J C D L ' 20$, August 1-5, 2020, Virtual Event, China. (C) 2020 Copyright is held by the owner/author(s). ACM ISBN 978-1-4503-7585-6/20/06.

DOI: https://doi.org/10.1145/3383583.3398632 application beyond simple POI (Point Of Interest) approaches? Typical POI approaches are merely pinpointing locations, leaving navigation and consideration of story flow to the users. We are planning to explore visualization through software prototypes and user studies. In order to guide the research, we defined the following scoping questions:

Q1: How to combine AR technology and location-based data for marking location order?

Q2: How can AR visualization reflect and navigate users to location-based data that narrates a story?

As a first step to explore these questions, we built a mobile prototype that provides Narrative Navigation through AR for location-based stories. Through the use of location-based AR, the users are guided and supported to navigate to the next story location. We visualize the story flow with reference to the user's physical location.

\section{PROTOTYPE \& USE CASE}

For our prototype, we first selected a dataset, then decided on the general flow of the application. The prototype is being used to explore different marker placements and to identify possible adjustments to indicate narrative order. Our location-based digital story uses cross-media elements to expand paper-based stories to connect with location-based information. Our prototype uses Unity $3 \mathrm{D}$, ARCore, and $\mathrm{C \#}$ as a script control language to realize its functionality, and was built for the Android mobile environment.

We here use a scenario in which a story about local trees is told in five ordered locations; visiting these five locations one after the other allows a user to experience the story in the order in which it is narrated (see story places 1 to 5 in Figure 1). The five elements represent five chapters respectively. We currently use a red marker (a stylized flag) to indicate the next location in the story, and bluecolored markers for all further locations, see Figures 2 and 3. We indicate two aspects within each marker: the distance between the story location and the user and the narrative order in which the locations should be visited. We use the height of the flags to indicate the order of a story and the order in which a user should visit each story point: the highest flag in the scene is the next location to be visited (indicated also in red). The lowest flag in a 
scene is, thus, the last location to be visited (with respect to the current viewing direction).

To illustrate our app, we created two scenarios referring to two possible user locations (indicated by the red dots in our map in Figure 1). A user is assumed to be standing at one of these red dots pointing their tablet (or phone) in the direction represented by the red arrow (see Figure 1). When using our prototype app standing at location 1 (see Figure 1) the user then sees the AR markers for the five story chapters superimposed on the camera view of the tablet/phone as shown in Figure 2. When standing in location 2, the user would see the screen as shown in Figure 3: only four locations are shown now as the users had already visited location 1 . The user can thus easily identify the next story chapter, the correct story flow, and the distance to the locations of the story chapters.

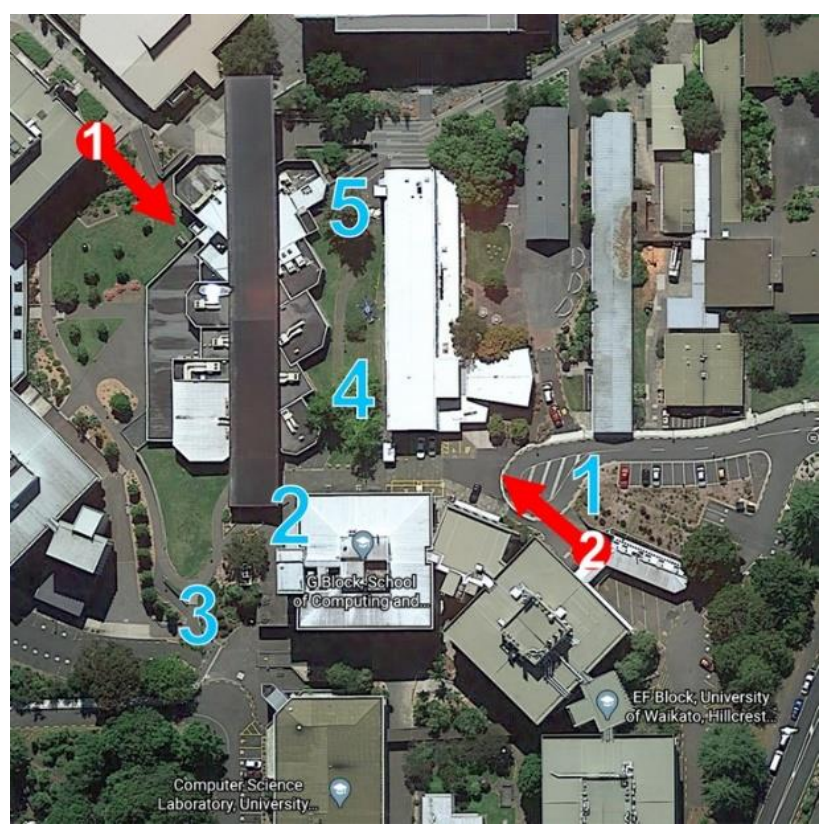

Figure 1: Five POI used in demonstration (@2020 Google)

\section{APP DEMONSTRATION}

We here briefly discuss the two consecutive scenes for the prototype (see Figures 2 and 3). In the situation shown in Figure 2, the first story location (1) is indicated by a red flag towards the top of the screen, and the flags of the next locations are displayed in decreasing heights. From left to right, the story chapters that are flagged are locations $5,1,4,2$, and 3 .

In our second scene, depicts scenario 2 whereby the user will have headed to and visited the location of the first chapter. Figure 3 then shows the Narrative Navigation from this second point. Now Chapter 2 is flagged in red as the next location to visit. The locations shown from left to right are: 3, 2, 4, 5; their descending order on screen indicates the story flow. From here the user would head to the top-flagged Chapter 2 location. A recording of this scenario walkthrough is available on our YouTube channel: https://bit.ly/30ysZ4K.

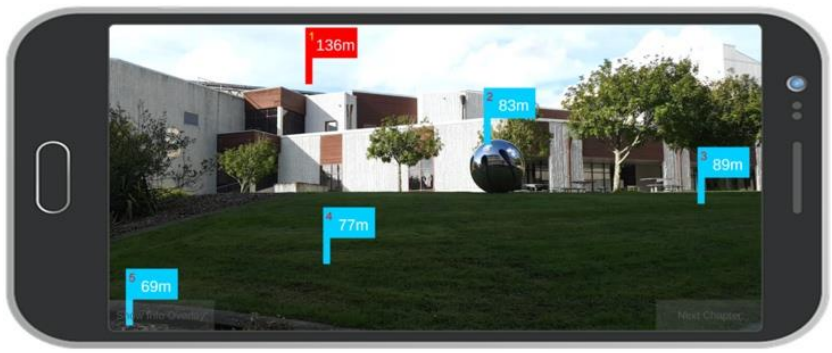

Figure 2: Scene 1 of the prototype

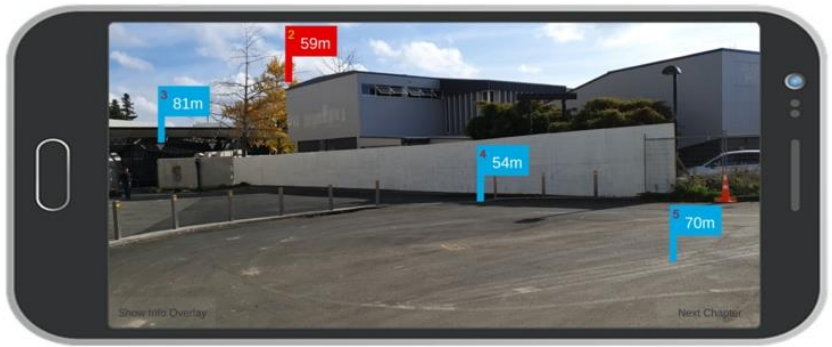

Figure 3: Scene 2 of the prototype

Using the prototype allowed us to observe that the story flags need to be shown stacked in relation to the user's view of the scene, not purely the location's terrain. Otherwise the flags may be located very high or low with respect to the user, depending on the surrounding terrain. This aspect needs to be explored further in future research.

\section{CONCLUSION \& LESSONS LEARNED}

We here introduced location-based markers to aid Narrative Navigation of a story. The prototype uses AR to help users to identify the next location in the story order. From our experimentation with this prototype, we learned that (1) users need to be alerted when they arrive at a location, (2) visited chapters need to be removed from the navigation, and (3) additional local navigation may be helpful to reach story points. Next, we plan to investigate the impact of terrain of the marker visualization.

\section{REFERENCES}

[1] Hinze, Annika and David Bainbridge, 2016. Location-triggered mobile access to a digital library of audio books using Tipple. International Journal on Digital Libraries, (2016), 339-365. DOI: https://doi.org/10.1007/s00799-015-0165-z

[2] Wither John, Allen Rebecca, Samanta Vids, et al. 2010. The Westwood Experience: Connecting story to locations via Mixed Reality. Paper presented at the 2010 IEEE International Symposium on Mixed and Augmented Reality - Arts, Media, and Humanities. 39-46. DOI: https://doi.org/10.1109/ISMARAMH.2010.5643295 